\title{
Anidulafungin: an evidence-based review of its use in invasive fungal infections
}

\author{
Susan L. Davis, Jose A. Vazquez
}

Henry Ford Hospital, Wayne State University College of Pharmacy, Wayne State University School of Medicine, Detroit, Michigan, USA

\begin{abstract}
Introduction: Anidulafungin is a new echinocandin antifungal agent with indications for use in esophageal candidiasis and candidemia. The mortality and morbidity associated with fungal infections in healthcare facilities necessitates the development of new treatment options for these diseases.

Aims: This review assesses the pharmacology and evidence for the use of anidulafungin in the treatment of serious fungal infections.

Evidence review: There is substantial evidence that anidulafungin is a potent antifungal agent with activity against a broad range of fungal species. Likewise, evidence supports that anidulafungin is a well-tolerated antifungal agent. Clinical studies provide sufficient evidence for regulatory approval for esophageal candidiasis and candidemia, and limited evidence suggests that anidulafungin may be superior to fluconazole for candidemia and invasive candidiasis. The introduction of anidulafungin into clinical practice adds a third option for therapy in the echinocandin class. Research into its efficacy in other fungal infections is ongoing, and further studies into the impact of anidulafungin on economic outcomes will be beneficial.
\end{abstract}

Place in therapy: Current evidence supports the use of anidulafungin in the management of candidemia, esophageal candidiasis, and invasive candidiasis, as demonstrated by the successful results in large multicenter clinical trials.

Core Evidence. 2008;2(4):241-249

Key words: anidulafungin, candidemia, candidiasis, echinocandins, evidence, review, treatment

Core evidence place in therapy summary for anidulafungin in candidiasis

\begin{tabular}{|lll|}
\hline Outcome measure & Evidence & Implications \\
\hline $\begin{array}{l}\text { Disease-oriented evidence } \\
\text { Microbiologic response }\end{array}$ & Clear & Achieved in the majority of patients with few episodes of invasive infection \\
$\begin{array}{l}\text { Patient-oriented evidence } \\
\text { Clinical response }\end{array}$ & Clear & Effective \\
Few drug-induced adverse events & Substantial & Safe and easy to use, and well tolerated, with low discontinuation rates \\
No drug-drug interactions & Clear & Safe and easy to use in patients on numerous medications \\
\hline
\end{tabular}




\section{Scope, aims, and objectives}

Anidulafungin is one of several drugs in the echinocandin class of antifungal agents. This evidence-based review focuses on the pharmacology and place in therapy for anidulafungin, the newest of the echinocandin antifungal agents. There have been several prior reviews on anidulafungin within the last few years (Murdoch \& Plosker 2004; Pfaller 2004; Raasch 2004; Vazquez 2005; Vazquez 2006a,b; Vazquez \& Sobel 2006). Most reviews, however, were published prior to the presentation of the data from the last two clinical trials performed with anidulafungin. In addition, this review utilizes evidence-based medical data in an attempt to identify anidulafungin's place in the antifungal armamentarium. Anidulafungin is the most recent addition to the echinocandin class of antifungals, which includes caspofungin and micafungin (Table 1).

\begin{tabular}{|c|c|c|c|}
\hline Indication & Anidulafungin & Caspofungin & Micafungin \\
\hline Esophageal candidiasis & $\begin{array}{l}\text { FDA } \\
\text { approved }\end{array}$ & $\begin{array}{l}\text { FDA } \\
\text { approved }\end{array}$ & $\begin{array}{l}\text { FDA } \\
\text { approved }\end{array}$ \\
\hline $\begin{array}{l}\text { Candidemia and other } \\
\text { forms of Candida infection } \\
\text { (intra-abdominal abscess } \\
\text { and peritonitis) }\end{array}$ & $\begin{array}{l}\text { FDA } \\
\text { approved }\end{array}$ & $\begin{array}{l}\text { FDA } \\
\text { approved }\end{array}$ & \\
\hline $\begin{array}{l}\text { Empirical therapy for } \\
\text { presumed fungal } \\
\text { infections in febrile } \\
\text { neutropenic patients }\end{array}$ & & $\begin{array}{l}\text { FDA } \\
\text { approved }\end{array}$ & \\
\hline $\begin{array}{l}\text { Invasive aspergillosis in } \\
\text { patients who are refractory } \\
\text { to or intolerant of other } \\
\text { therapies }{ }^{b}\end{array}$ & & $\begin{array}{l}\text { FDA } \\
\text { approved }\end{array}$ & \\
\hline $\begin{array}{l}\text { Prophylaxis of Candida } \\
\text { infections in patients } \\
\text { undergoing hematopoietic } \\
\text { stem cell transplantation }\end{array}$ & & & $\begin{array}{l}\text { FDA } \\
\text { approved }\end{array}$ \\
\hline \multicolumn{4}{|c|}{$\begin{array}{l}\text { aaCaspofungin is also indicated for treatment of pleural space infections. } \\
\text { bCaspofungin has not been studied as initial therapy for invasive aspergillosis. } \\
\text { FDA, Food and Drug Administration. }\end{array}$} \\
\hline
\end{tabular}

\section{Methods}

Searches of English language medical literature were conducted between May 1 and May 5, 2007, and November 1-9, 2007 in the following databases:

- PubMed, http://www.ncbi.nlm.nih.gov/entrez/query.fcgi, 1966 to date

- American Society for Microbiology abstract database, http://www.abstracts2view.com/icaac

- http://www.antimicrobe.org

- http://www.doctorfungus.org
- http://www.merck.com/product/usa/pi_circulars/c/cancidas/ cancidas_pi.pdf

- http://www.pfizer.com/pfizer/download/uspi_eraxis.pdf

- http://www.astellas.us/docs/mycamine.pdf

The search terms utilized included "anidulafungin," "caspofungin," "micafungin," "Eraxis," "Cancidas," "Mycamine," "echinocandins," "candidemia," "candidiasis," "esophageal candidiasis," "LY 303366," and "VER-002." The literature search results are shown in Table 2.

\section{Table 2 | Evidence base included in the review}

\begin{tabular}{|lcc|}
\hline Category & \multicolumn{2}{c}{ Number of records } \\
\cline { 2 - 3 } & Full papers & Abstracts \\
\hline Initial search & 89 & 11 \\
records excluded & 33 & 6 \\
records included & 56 & 5 \\
Level 1 clinical evidence & 2 & 0 \\
Level 2 clinical evidence & 2 & 2 \\
Level $\geq 3$ clinical evidence & 4 & 2 \\
trials other than RCT & 46 & 1 \\
case studies & 2 & 0 \\
Economic evidence & 0 & 0 \\
\hline For definitions of levels of evidence, see Editorial Information on inside back cover or on \\
Core Evidence website (http://www.coremedicalpublishing.com). \\
RCT, randomized controlled trial.
\end{tabular}

\section{Disease overview}

Invasive fungal infections are of increasing importance in hospitalized patients, both with and without underlying immunocompromise. Over the past decade the frequency and diversity of invasive fungal infections has dramatically increased. Infections due to Candida species are currently the fourth most common cause of bloodstream infections (Wisplinghoff et al. 2004), while invasive aspergillosis has become the most common serious mold infection in immunocompromised hosts (Denning et al. 1998). While infections with Aspergillus species present significant diagnostic and therapeutic challenges with considerable morbidity, mortality, and cost, it is clear that infections due to Candida spp. are much more commonly observed in hospitalized patents. Serious Candida infections may range from primary bloodstream infections and catheterrelated infections to disseminated candidiasis, where candidemia may not be apparent. The attributable mortality rates are difficult to estimate; crude mortality rates range from $30-60 \%$ (Zaoutis et al. 2005).

\section{Current therapy options}

Recent literature has shown that early initiation of antifungal therapy can improve patient outcomes, and considerable research is ongoing to define optimal therapy (Garey et al. 2006; Davis et al. 2007). 
Guidelines for the management of candidemia and candidiasis have been published by the Mycosis Study Group and Infectious Disease Society of the Americas and can be accessed via the website www.idsociety.org (Pappas et al. 2004). For several decades, amphotericin B was the only antifungal agent available for the treatment of systemic fungal infections. However, over the past 10-15 years several new classes of antifungals, specifically the azoles (fluconazole, itraconazole, voriconazole, posaconazole) and the echinocandins (caspofungin, micafungin, anidulafungin) are now available to manage these severe infections. For the past decade, fluconazole has been the main antifungal used to treat candidal infections in most types of patients. Unfortunately, over the past decade, two major changes have occurred that have precipitated a change in the management of candidal infections. The first change has been a well-documented shift in the Candida species causing candidemia and candidiasis, from the susceptible Candida albicans, to the less susceptible nonalbicans Candida (NAC) species. Of the NAC species, C. glabrata, C. parapsilosis, C. tropicalis, and C. krusei constitute the bulk of infections. These NAC species are important because they have varying susceptibilities to azoles and amphotericin $\mathrm{B}$. The second change has been the development of fluconazole resistance in some of the NAC species, especially $C$. glabrata. The changing epidemiology of fungal infections coupled with the significant mortality and cost associated with the treatment of fungal infection has driven the development of new antifungal agents. Although new guidelines are under development, the current recommendation is to initiate antifungal therapy with either fluconazole, one of the echinocandins, or voriconazole, depending on the species of Candida identified in the patient (Pappas et al. 2004). In patients that are hemodynamically unstable, neutropenic, or infected with either C. glabrata or C. krusei the echinocandins are the preferred antifungal agents.

\section{Unmet needs}

Despite the availability of several new antifungal agents, the attributable mortality rates associated with candidemia and invasive candidiasis are generally between $30-40 \%$, and have remained unchanged for the past decade. In addition, because of the extensive amount of fluconazole use in clinical practice over the past 10-15 years, we now have to recognize the increased frequency of azole resistance in the NAC species, especially in C. glabrata. These two reasons are why the echinocandins (caspofungin, micafungin, and anidulafungin) have become the preferred antifungal agents in seriously ill patients with disseminated fungal infections.

\section{Anidulafungin pharmacology}

\section{Chemistry and mechanism}

Echinocandin antifungals were first discovered in the 1970s, and named the pneumocandins for their activity against Pneumocystis (Denning 1997). Approved by the FDA in 2006, anidulafungin was the first echinocandin discovered; however, caspofungin and micafungin were introduced into the market in 2001 and 2005, respectively. Anidulafungin is derived from echinocandin $B_{0}$, which is produced by $A$. nidulans. Anidulafungin's structure consists of an amphiphilic hexapeptide linked to an alcoxytriphenyl side chain. It is this $\mathrm{N}$-linked acyl lipid side chain that differs between the echinocandins, and accounts for some of the differences in their properties (Kurtz \& Rex 2001). Drugs in the echinocandin class of antifungals inhibit cell wall synthesis, causing cell wall damage through noncompetitive inhibition of beta-(1,3)-glucan-synthase (Douglas 2001). Depletion of beta$(1,3)$-glucan, a vital component of the fungal cell wall structure, leads to osmotic cell lysis.

\section{Pharmacodynamics}

Anidulafungin demonstrates in-vitro activity against many clinically relevant fungal species including a broad range of Candida species, such as those strains resistant to fluconazole or amphotericin B. In-vitro susceptibility assays reveal a decreased activity against $C$. parapsilosis, although no direct correlation between minimum inhibitory concentration (MIC) and clinical outcome has been established for echinocandins (Table 3) (Marco et al. 1998; Uzun et al. 1997; Zhanel et al. 1997; Wiederhold et al. 2003; Pfaller et al. 2005; Pfaller et al. 2006). Furthermore,

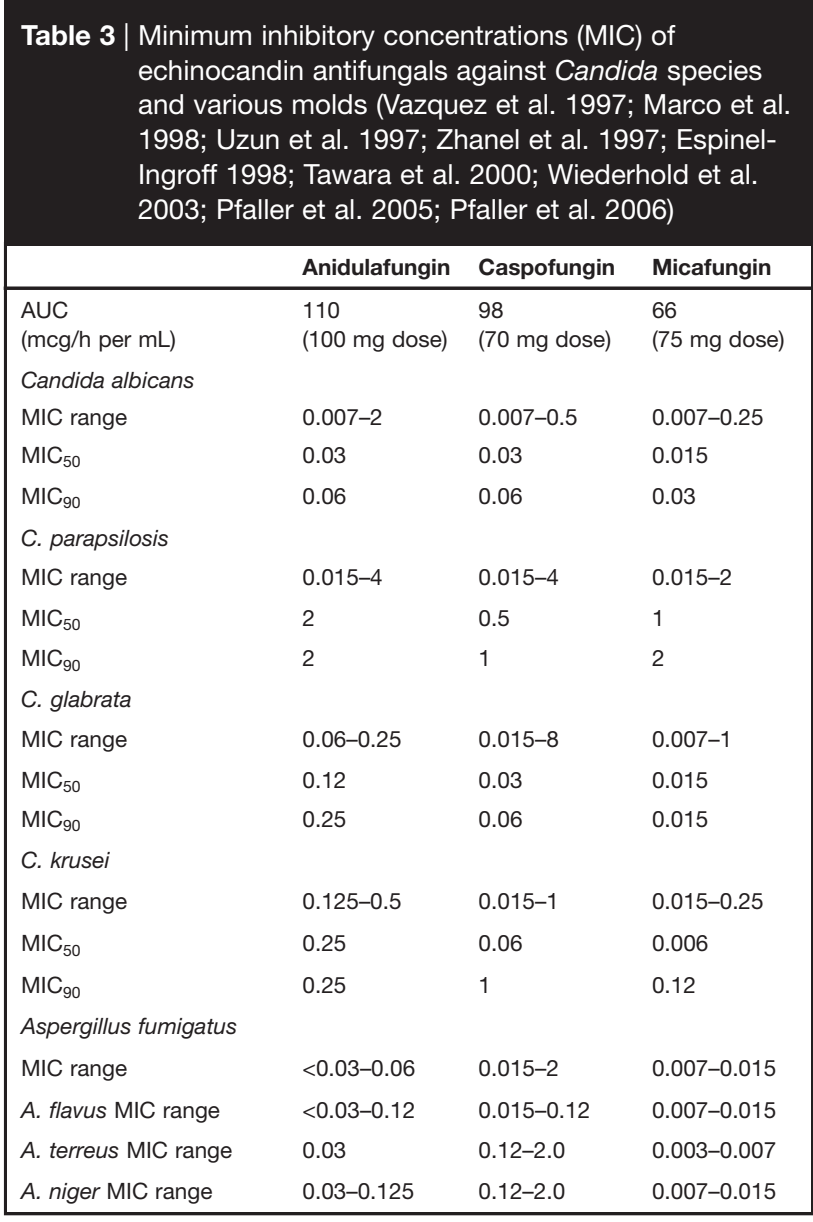


anidulafungin also has proven in-vitro activity against fluconazole- and itraconazole-resistant Candida species recovered from HIV-positive individuals (Chavez et al. 1999; Cuenca-Estrella et al. 2000; Pfaller et al. 2005). Anidulafungin has also demonstrated in-vitro activity against Aspergillus species including $A$. fumigatus, $A$. flavus, $A$. niger, and $A$. terreus, along with activity against many of the emerging molds such as Bipolaris spicifera, Exophiala jeanselmei, Fonsecaea pedrosoi, Madurella mycetomatis, Penicillium marneffei, Phialophora verrucosa, Pseudallescheria boydii, Sporothrix schenckii, and Wangiella dermatitidis (Espinel-Ingroff 1998; Odabasi et al. 2004).

In addition, anidulafungin has demonstrated additive effects in combination with amphotericin B against Aspergillus and Fusarium species, and potential synergistic activity in combination with itraconazole or voriconazole against Aspergillus species (Philip A et al. 2005; Vazquez 2007).

The pharmacodynamic activity of anidulafungin (like other echinocandins) differs between organisms. Against Aspergillus, anidulafungin exerts a fungistatic effect in vivo, and demonstrates fungicidal activity against Candida species in vitro and in vivo. Against Candida species, the effect of echinocandins has been shown to be related to concentration and area under the concentration-time curve (Petraitiene et al. 1999; Louie et al. 2005). Additionally, an Eagle-like effect (paradoxical increase in fungal growth at higher concentrations) has been observed in yeast and filamentous fungi; however additional investigation into the mechanism and clinical significance of this relationship is warranted (Clemons \& Stevens 2006; Wiederhold et al. 2004). In-vitro assays of anidulafungin in the presence of human serum suggest that serum binding reduces the activity of echinocandins against glucan synthase (Paderu et al. 2007). The clinical significance of the high protein binding is currently not known.

\section{Resistance}

The definition of echinocandin resistance is yet to be defined, but recent Clinical and Laboratory Standards Institute guidelines define a susceptible isolate with an MIC of $<2 \mathrm{mcg} / \mathrm{mL}$ as susceptible, and isolates with MICs to echinocandins of $>2 \mathrm{mcg} / \mathrm{mL}$ as nonsusceptible. To date, reduced echinocandin susceptibility remains uncommon, although most of the research to date has evaluated caspofungin susceptibility and reduced susceptibility. There have been several reports describing crossresistance of echinocandins in different Candida species after prolonged exposures to echinocandins (Hakki et al. 2006; Laverdiere et al. 2006). In addition, in one series, although the activity was compromised, anidulafungin retained greater activity among $C$. glabrata isolates with reduced caspofungin susceptibility (Cota et al. 2006). In a recent case report by Moudgal et al., the authors described the first case of a multiechinocandin-resistant $C$. parapsilosis infection in a patient with refractory prosthetic valve endocarditis (Moudgal et al. 2005). Interestingly enough, all of the isolates that demonstrated reduced susceptibility to caspofungin and micafungin remained susceptible to anidulafungin. More recently, Chen et al. (2005) described 31 isolates of $C$. parapsilosis recovered from burn unit patients that displayed reduced susceptibility to micafungin and or caspofungin, but retained their susceptibility to anidulafungin. The mechanism of this cross-reduced echinocandin susceptibility is currently unknown.

Although the mechanisms of resistance to the echinocandins are not yet fully understood, there are several mechanisms that have been recently elucidated and reported. The most commonly described mechanisms involve the FKS1 and FKS2 genes that encode for the catalytic subunit of beta-(1,3)-glucan synthase (Douglas 2001; Douglas et al. 1994). Several investigators have already described mutations in two different "hotspots" of the FKS1 gene that have been shown to confer resistance to caspofungin in C. albicans, C. glabrata, and C. guilliermondii (Park et al. 2005; Balashov 2006; Katiyar 2006; Miller 2006). Other purported resistance mechanisms are the overproduction of beta-(1,3)-glucan synthase and the expression of efflux pumps (Osherov et al. 2002; Ghannoum 2005). Echinocandins have no activity against Cryptococcus neoformans, possibly related to impaired binding to a variation of glucan synthase in this organism (Maligie \& Selitrennikoff 2006).

\section{Pharmacokinetics}

Like other echinocandins, oral bioavailability of anidulafungin is less than $10 \%$, and thus is only available for parenteral administration. Protein binding was originally reported to be 84\% (Wiederhold \& Lewis 2003); however, it is now believed to be approximately $98 \%$, which is consistent with the other agents in the class (Eraxis prescribing information). Anidulafungin demonstrates a large volume of distribution of 30-50L, and a terminal elimination half-life of 25 hours, following a biexponential decay. Steady-state concentrations are achieved on the first day of therapy after administration of a loading dose twice the normal daily dose. Anidulafungin is not metabolized by the liver, but undergoes slow chemical degradation by peptidases. An intermediate metabolite is formed by the opening of the hexapeptide ring, and this is then metabolized in plasma to tertiary degradation products. Approximately $10 \%$ of anidulafungin is eliminated unchanged via the biliary system. Since the drug is not degraded by hepatic enzymes, there are no interactions with the cytochrome P450 system.

Few data exist on anidulafungin in pediatric patients; however, an evaluation of safety and pharmacokinetics was conducted in a group of neutropenic children 2-17 years of age. A total of 24 patients were administered $0.75 \mathrm{mg} / \mathrm{kg}$ per day or $1.5 \mathrm{mg} / \mathrm{kg}$ per day anidulafungin based on total body weight, and pharmacokinetic parameters were determined. The study excluded children with hepatic or renal disease. The investigators determined that pediatric patients receiving $0.75 \mathrm{mg} / \mathrm{kg}$ per day or $1.5 \mathrm{mg} / \mathrm{kg}$ per day achieved similar anidulafungin concentrations to adults receiving 50 or $100 \mathrm{mg} /$ day, respectively (Benjamin 2006). 


\section{Clinical evidence with anidulafungin}

\section{Esophageal candidiasis}

The efficacy of anidulafungin in treating esophageal candidiasis was studied in a randomized, double-dummy, controlled clinical trial that included 601 patients with endoscopically proven esophageal candidiasis (Krause 2004a) (Table 4). A total of 300 patients received an intravenous loading dose of anidulafungin $100 \mathrm{mg}$, followed by $50 \mathrm{mg} /$ day, and 301 patients received oral fluconazole $100 \mathrm{mg} /$ day. Treatment was continued for 14-21 days (a minimum of 7 days after resolution of symptoms). Of the 504 patients who completed therapy, 242 out of 249 (97.2\%) who received anidulafungin and 252 out of 255 (98.8\%) who received fluconazole demonstrated endoscopic evidence of cure or improvement, indicating that anidulafungin is at least as efficacious as fluconazole in the treatment of esophageal candidiasis. The one difference between treatment groups was the relapse rate. At the 2-week follow-up, the relapse rate was $35.6 \%$ for anidulafungin and $10.5 \%$ for fluconazole. Although the relapse rate was high with anidulafungin, a similar relapse rate was also seen in patients with oropharyngeal candidiasis (OPC) and/or esophageal candidiasis treated with caspofungin (Arathoon et al. 2002). The cause of this higher relapse rate associated with echinocandin use when compared with fluconazole is unknown.

Anidulafungin is also effective in managing patients with fluconazole-refractory mucosal candidiasis. Anidulafungin was evaluated in a small, open-label clinical trial in patients with refractory oropharyngeal or esophageal candidiasis who had failed at least 14 days' treatment with fluconazole $>200$ mg/day (Vazquez et al. 2007). In an interim analysis of 18 patients enrolled in the study, 17 had a diagnosis of AIDS (CD4 cell count $<50$ cells $/ \mathrm{mm}^{3}$ ). At the end of therapy, 17 out of 18 patients had a successful clinical response, and 10 out of 11 patients with esophageal candidiasis were considered endoscopic successes. Clinical response was defined as cure (absence of symptoms and requiring no additional therapy), improvement (decrease in severity when compared to baseline and no further need for antifungals), or failure. In addition, at the end of therapy, if patients had esophageal candidiasis at baseline, the endoscopic response was also evaluated and classified as cure, improvement, or failure. Furthermore, anidulafungin was well tolerated with only one serious adverse event (rash). Anidulafungin appears to be a promising treatment regimen for azole-refractory mucosal candidiasis.

\section{Candidemia and invasive candidiasis}

A phase II, open-label, dose-ranging study was conducted in 123 patients with candidemia and signs or symptoms of active infection (Krause et al. 2004b) (Table 4). Participants received anidulafungin at dosages of 50,75 , or $100 \mathrm{mg} /$ day. Success was defined as both a clinical and microbiological success, as documented by resolved clinical manifestations and sterilization of blood cultures. Anidulafungin was successful in 73 out of the 83 evaluable patients at the end of antifungal therapy (84\%, 90\%, and $89 \%$, respectively, for the different dosage regimens). At the 2-week follow-up period, the corresponding success rates in these very ill patients were 72,85 , and $83 \%$, favoring the two higher dosages. Anidulafungin was well tolerated at all dosages and adverse events were uncommon. Adverse events considered to be treatment-related were reported in $<5 \%$ of patients in any dose group, with the exception of hypokalemia, which was reported in $10 \%$ of patients in the $50 \mathrm{mg}$ dose group. Overall, the most commonly reported events were hypotension (13\%), vomiting (13\%), constipation (11\%), and nausea (11\%). There was no dose-response relationship with respect to the frequency of adverse events.

Table 4 | Clinical trials evaluating anidulafungin in candidemia and esophageal candidiasis

\begin{tabular}{|c|c|c|c|c|c|c|}
\hline Disease & $\begin{array}{l}\text { No. patients } \\
\text { enrolled }\end{array}$ & $\begin{array}{l}\text { Treatment and dosage } \\
\text { regimen }\end{array}$ & Outcome & Adverse events & $\begin{array}{l}\text { Level of } \\
\text { evidence }\end{array}$ & Reference \\
\hline \multirow[t]{2}{*}{$\begin{array}{l}\text { Esophageal } \\
\text { candidiasis }\end{array}$} & \multirow[t]{2}{*}{36} & $\begin{array}{l}\text { Afgn } 50 \mathrm{mg} \text { LD, followed by } \\
25 \mathrm{mg} / \mathrm{d}\end{array}$ & \multirow[t]{2}{*}{$\begin{array}{l}\text { Endoscopic response rates } \\
81 \% \text { vs } 85 \%\end{array}$} & \multirow[t]{2}{*}{ N/A } & \multirow[t]{2}{*}{3} & \multirow[t]{2}{*}{ Brown et al. 2000} \\
\hline & & $\begin{array}{l}\text { Afgn } 70 \mathrm{mg} \text { LD, followed by } \\
50 \mathrm{mg} / \mathrm{d}\end{array}$ & & & & \\
\hline \multirow[t]{2}{*}{$\begin{array}{l}\text { Esophageal } \\
\text { candidiasis }\end{array}$} & \multirow[t]{2}{*}{601} & $\begin{array}{l}\text { Afgn } 100 \mathrm{mg} \text { LD, followed } \\
\text { by } 50 \mathrm{mg} / \mathrm{d}\end{array}$ & \multirow{2}{*}{$\begin{array}{l}\text { Endoscopic response rates: } \\
242 / 249(97 \%) \text { vs } 252 / 255 \\
(98.8 \%)\end{array}$} & \multirow[t]{2}{*}{$9.3 \%$ vs $12 \%$} & \multirow[t]{2}{*}{2} & \multirow[t]{2}{*}{ Krause et al. 2004a } \\
\hline & & $\begin{array}{l}\text { Flz } 100 \mathrm{mg} \text { LD, followed by } \\
100 \mathrm{mg} / \mathrm{d}\end{array}$ & & & & \\
\hline \multirow{3}{*}{$\begin{array}{l}\text { Candidemia and } \\
\text { invasive candidiasis }\end{array}$} & \multirow[t]{3}{*}{123} & Afgn $50 \mathrm{mg} / \mathrm{d}$ & \multirow{3}{*}{$\begin{array}{l}\text { Global response rates } 72 \% \\
\text { vs } 85 \% \text { vs } 83 \%\end{array}$} & \multirow[t]{3}{*}{$<5 \%$ in all three groups } & \multirow[t]{3}{*}{3} & \multirow[t]{3}{*}{ Krause et al. 2004b } \\
\hline & & Afgn $75 \mathrm{mg} / \mathrm{d}$ & & & & \\
\hline & & Afgn $100 \mathrm{mg} / \mathrm{d}$ & & & & \\
\hline \multirow[t]{2}{*}{$\begin{array}{l}\text { Candidemia and } \\
\text { invasive candidiasis }\end{array}$} & \multirow[t]{2}{*}{245} & $\begin{array}{l}\text { Afgn } 200 \mathrm{mg} \text { LD, followed } \\
\text { by } 100 \mathrm{mg} / \mathrm{d}\end{array}$ & \multirow[t]{2}{*}{$\begin{array}{l}\text { Global response rate at end } \\
\text { of i.v. } 75.6 \% \text { vs } 60.2 \%\end{array}$} & \multirow[t]{2}{*}{$\begin{array}{l}\text { Comparable in both } \\
\text { groups }\end{array}$} & \multirow[t]{2}{*}{2} & \multirow[t]{2}{*}{ Reboli et al. 2007} \\
\hline & & $\begin{array}{l}\text { Flz } 800 \mathrm{mg} \text { LD, followed by } \\
400 \mathrm{mg} / \mathrm{d}\end{array}$ & & & & \\
\hline \multirow{2}{*}{$\begin{array}{l}\text { Antifungal-refractory } \\
\text { mucosal candidiasis }\end{array}$} & \multirow[t]{2}{*}{19} & \multirow{2}{*}{$\begin{array}{l}\text { Afgn } 200 \mathrm{mg} \text { LD, followed } \\
\text { by } 100 \mathrm{mg} / \mathrm{d}\end{array}$} & Clinical response $18 / 19$ & Nausea/vomiting 20\% & \multirow[t]{2}{*}{3} & \multirow[t]{2}{*}{ Vazquez et al. 2007} \\
\hline & & & Endoscopic response $11 / 12$ & Hypokalemia 10\% & & \\
\hline
\end{tabular}


In a recently completed phase III, multicenter, double-blind, randomized clinical trial, a $100 \mathrm{mg}$ dose of anidulafungin was compared with a $400 \mathrm{mg}$ dose of fluconazole in patients with candidemia and/or invasive candidiasis (Reboli et al. 2007) (Table 4). A total of 261 patients were enrolled in this pivotal clinical trial, 256 were included in the intent-to-treat population, and 245 in the modified intent-to-treat population. In the study 127 patients received at least one dose of anidulafungin while 118 patients received fluconazole. The primary analysis was performed at the end of intravenous therapy (day 10). Success was defined as both a clinical and microbiological success, as documented by resolved clinical manifestations and sterilization of blood cultures. A successful outcome was achieved in $76 \%$ of patients who received anidulafungin compared with a $60 \%$ success in patients who received fluconazole. The difference between groups was $15.4 \%$, with a $95 \%$ confidence interval of 3.9 to 27.0. Additionally, at the 2-week follow-up period, a similar treatment difference in response was observed, with a success rate of $65 \%$ and $49 \%$, respectively. Compared with those subjects who received fluconazole, anidulafungin recipients had a higher rate of success for every Candida species except for C. parapsilosis. However, the total number of patients with C. parapsilosis was only $24(10 \%)$ of the total population (10 patients in the anidulafungin arm and 14 patients in the fluconazole arm), thus making it difficult to make any definitive comments regarding the treatment differential. In addition, it was not a statistically significant difference. One of the limitations to this study was associated with the fact that the evidence for superiority of anidulafungin over fluconazole was decreased when data from the highest enrolling site were excluded from the analysis. However, statistical analysis was not able to demonstrate a "center effect," and global success rates were $73 \%$ in the anidulafungin arm compared with $61 \%$ in the fluconazole arm (treatment difference of $12.1 \% ; 95 \% \mathrm{Cl}-1.1,25.3$ ).

Overall, the adverse event and tolerability profiles were similar between the two treatment groups. Treatment-related adverse events were only observed in two patients in each arm, with one case of seizures and one case of atrial fibrillation in the anidulafungin arm. In general, most of the adverse events reported were due to the underlying disease state and not the study drugs. In addition, the mortality rate from all causes was found to be $31 \%$ in the fluconazole arm compared with $23 \%$ in the anidulafungin arm. Although it was an $8 \%$ difference, it did not reach statistical difference.

\section{Safety}

In clinical studies documenting over 600 patients, anidulafungin has been well tolerated compared with adverse effects seen with other antifungals. The most common adverse effects reported include headache and nausea. In a few cases mild, reversible elevations of liver function tests have been seen. With rapid intravenous administration some flushing, nausea, and dyspnea were reported; however, infusion-related reactions quickly subside and can be greatly reduced with slower infusion rates. No specific monitoring parameters are routinely recommended in patients receiving anidulafungin (Krause et al. 2004ab; Reboli et al. 2007).

\section{Drug interactions}

In studies performed to date, anidulafungin has not been associated with any significant drug-drug interactions. Concurrent dosing with cyclosporine (a CYP3A4 substrate) following anidulafungin $100 \mathrm{mg} /$ day demonstrated no significant alteration in pharmacokinetics of either drug (Dowell et al. 2005). No changes in disposition were observed with concomitant administration of anidulafungin and voriconazole (Dowell et al. 2005) and liposomal Amphotericin B. Similarly, no interactions have been identified between anidulafungin and any substrates, inhibitors, or inducers of cytochrome P450 enzymes, including rifampin, efavirenz, or nevirapine.

While few postmarketing data have been published on the safety of anidulafungin, a small study provides information on the potential for disulfiram-like reaction when anidulafungin and its alcoholcontaining diluent is administered with concomitant metronidazole. A prospective cohort was followed for potential disulfiram-like reaction, defined as having at least one symptom from each category of general disorder, gastrointestinal, and neurologic symptoms. Of 44 patients receiving the combination of agents, none experienced a disulfiram-like reaction (Hurren et al. 2007).

\section{Economic evidence and resource utilization}

The treatment of candidemia has been associated with an attributable cost of nearly \$US40 000 per case and greater than 10 additional days of hospitalization (Zaoutis et al. 2005).

There is no economic evidence for the use of anidulafungin over other echinocandins or azoles in the treatment of candidemia and or invasive candidiasis. However, the fungicidal activity in vitro of anidulafungin compared with the fungistatic activity of the azoles, and the fact that the pivotal candidemia trial demonstrated statistical superiority of anidulafungin over fluconazole along with a decrease in mortality, suggest that the use of anidulafungin over fluconazole in patients with candidemia may prove beneficial. Cost-benefit and cost-effectiveness studies still need to be done comparing these two classes of antifungals.

\section{Dosage, administration, and formulations}

The FDA-approved dose of anidulafungin is based on the indication. For esophageal candidiasis, it is given as a $100 \mathrm{mg}$ intravenous loading dose, followed by $50 \mathrm{mg}$ intravenously once daily. For the treatment of candidemia and systemic candidiasis, a $200 \mathrm{mg}$ loading dose is given on day one, followed by $100 \mathrm{mg}$ daily. Clinical need for dose escalation has not been reported, but a one-time dose of $260 \mathrm{mg}$, the maximum dose studied, was well tolerated (Thye et al. 2001). The maximum cumulative dose in studies without additional adverse effects reported was daily doses of $100 \mathrm{mg}$ continued over 90 days (Dowell et al. 2004). Unlike other echinocandins, no adjustments of anidulafungin doses are necessary for either renal or hepatic insufficiency. The drug is a large molecule and is not well dialyzable; however, specific dosing recommendations in dialysis or continuous renal replacement therapy have not been established. 
Like other currently available echinocandins, anidulafungin is available only in intravenous preparations, owing to its large molecular weight and low oral bioavailability. Anidulafungin is insoluble in water, and is reconstituted in $20 \% \mathrm{w} / \mathrm{w}$ dehydrated alcohol in water for intravenous administration. After reconstitution in the manufacturer-provided solution, it can be diluted with either $0.9 \%$ sodium chloride or $5 \%$ dextrose and should be administered within 24 hours. A maximum infusion rate of $1.1 \mathrm{mg} / \mathrm{minute}$ is recommended to reduce the likelihood of infusion-related reactions.

\section{Place in therapy}

The safety and efficacy of anidulafungin, plus its novel pharmacokinetics, make it a suitable alternative antifungal for therapy of mucocutaneous candidiasis, candidemia, and invasive candidiasis. It provides a broad spectrum of activity with proven efficacy against a wide array of Candida species, including those that are azole- or polyene-resistant, and has also demonstrated activity against Aspergillus species.

Anidulafungin appears to have several advantages over other antifungal drugs. For example, it does not require dosing adjustments in patients based on age, gender, weight, disease state, concomitant drug therapy, or renal or hepatic insufficiency. In addition, because of its lack of hepatic metabolism and lack of P450 interaction, it does not have any drug-drug interactions. Furthermore, because of its unique mechanism of action, anidulafungin does not induce cross-resistance with other classes of antifungals.

It is the first antifungal agent that has demonstrated statistical superiority in a comparative clinical trial evaluating therapy for candidemia. Results of current studies warrant further clinical trials evaluating the use of anidulafungin as either primary or preemptive therapy for invasive fungal infections, such as candidemia and invasive candidiasis and aspergillosis. Moreover, because of its lack of adverse drug interactions and the possibility of in-vitro additive or synergistic activity with other antifungals, the role of anidulafungin in combination with other antifungals should be further explored in the management of some of the high mortality-associated mold infections, such as aspergillosis.

In summary, anidulafungin shows excellent promise and should be a valuable new antifungal agent with a novel mechanism of action. Completed clinical studies have demonstrated that anidulafungin should prove useful as a first-line agent for treating a range of serious fungal infections, including mucocutaneous candidiasis, candidemia, invasive candidiasis, and azolerefractory mucosal candidiasis. However, although it has in-vitro activity against Aspergillus species and other molds, its usefulness against serious mold infections such as aspergillosis, either as monotherapy or in combination, is currently unknown.

\section{Acknowledgments}

Dr Vazquez has received research grants from Pfizer, Merck, Schering Plough, Targenta, J\&J, and Basilea Pharmaceutica Ltd.

\section{References}

Arathoon EG, Gotuzzo E, Noriega LM, Berman RS, DiNubile MJ, Sable CA. Randomized, double-blind, multicenter study of caspofungin versus amphotericin B for treatment of oropharyngeal and esophageal candidiases. Antimicrob Agents Chemother. 2002;46:451-457.

Balashov SV, Park S, Perlin DS. Assessing resistance to the echinocandin antifungal drug caspofungin in Candida albicans by profiling mutations in FKS1. Antimicrob Agents Chemother. 2006;50:2058-2063.

Benjamin DK, Driscoll T, Seibel NL, et al. Safety and pharmacokinetics of intravenous anidulafungin in children with neutropenia at high risk for invasive fungal infections. Antimicrob Agents Chemother. 2006;50:632-638.

Brown GL, White RJ, Turik M. Phase II, randomized, open label study of two intravenous dosing regimens of $\mathrm{V}$-echinocandin in the treatment of esophageal candidiasis. In: Program and abstracts of the 40th Interscience Conference on Antimicrobial Agents and Chemotherapy. Toronto, Ontario, Canada, Sept 17-20, 2000. Abstract 1106.

Chavez M, Bernal S, Valverde A, Gutierrez MJ, Quindós G, Martin Mazuelos E. In vitro activity of voriconazole (UK-109,496), LY 303366 and other antifungal agents against oral Candida spp. isolates from HIV-infected patients. J Antimicrob Chemother. 1999;44:697-700.

Chen A, Buhari M, Boikov D, et al. The complex epidemiology of Candida colonization in a tertiary care burn unit. In: Program and Abstracts of the 45th Interscience Conference on Antimicrobial Agents and Chemotherapy, Washington DC, December 16-19, 2005. Abstract M-987.

Clemons KV, Stevens DA. Animal models of Aspergillus infection in preclinical trials, diagnostics and pharmacodynamics: What can we learn from them? Med Mycol. 2006;44:119-126.

Cota J, Carden M, Graybill JR, Najvar LK, Burgess DS, Wiederhold NP. In vitro pharmacodynamics of anidulafungin and caspofungin against Candida glabrata isolates, including strains with decreased caspofungin susceptibility. Antimicrob Agents Chemother. 2006;50:3926-3928.

Cuenca-Estrella M, Mellado E, Díaz-Guerra TM, Monzón A, Rodriguez-Tudela JL. Susceptibility of fluconazole-resistant clinical isolates of Candida spp. to echinocandin LY303366, itraconazole, and Amphotericin B. J Antimicrob Chemother. 2000;46:475-477.

Davis SL, Vazquez JA, McKinnon PS. Epidemiology, risk factors, and outcomes of Candida albicans versus non-albicans candidemia in nonneutropenic patients. Ann Pharmacother. 2007;41:568-573

Denning DW, Marinus A, Cohen J, et al. An EORTC multicentre prospective survey of invasive aspergillosis in haematological patients: diagnosis and therapeutic outcome. EORTC Invasive Fungal Infections Cooperative Group. J Infect. 1998;37:173-180.

Denning DW. Echinocandins and pneumocandins-a new antifungal class with a novel mode of action. J Antimicrob Chemother. 1997;40:611-614.

Douglas CM, Foor F, Marrinan JA, et al. The Saccharomyces cerevisiae FKS1 (ETG1) gene encodes an integral membrane protein which is a subunit of 1,3-beta-D-glucan synthase. Proc Natl Acad Sci U S

A.1994;91:12907-12911.

Douglas CM. Fungal beta(1,3)-D-glucan synthesis. Med Mycol. 2001;(39 Suppl 1):55-66.

Dowell JA, Knebel W, Ludden T, Stogniew M, Krause D, Henkel T. Population pharmacokinetic analysis of anidulafungin, an echinocandin antifungal. $\underline{J}$ Clin Pharmacol. 2004;44:590-598.

Dowell JA, Schranz J, Baruch A, Foster G. Safety and pharmacokinetics of coadministered voriconazole and anidulafungin. J Clin Pharmacol. 2005;45:1373-1382.

Dowell JA, Stogniew M, Krause D, Henkel T, Weston IE. Assessment of the safety and pharmacokinetics of anidulafungin when administered with cyclosporine. J Clin Pharmacol. 2005;45:227-233.

Espinel-Ingroff A. Comparison of In vitro activities of the new triazole SCH56592 and the echinocandins MK-0991 (L-743,872) and LY303366 against opportunistic filamentous and dimorphic fungi and yeasts. $\underline{J}$ Clin Microbiol. 1998;36:2950-2956. 
Garey KW, Rege M, Pai MP, et al. Time to initiation of fluconazole therapy impacts mortality in patients with candidemia: a multi-institutional study. Clin Infect Dis. 2006;43:25-31.

Ghannoum MA, Chandra J, Mukherjee PK, Chen A, Buhari M, Vazquez J. Emergence of a multi-echinocandin resistant Candida parapsilosis. In: Program and Abstracts of the 45th Interscience Conference on Antimicrobial Agents and Chemotherapy. Washington DC, Dec 16-19, 2005. Late breaker.

Hakki M, Staab JF, Marr KA. Emergence of a Candida krusei isolate with reduced susceptibility to caspofungin during therapy. Antimicrob Agents Chemother. 2006;50:2522-2524.

Hurren JR, Davis SL, Vazquez JA. What is the risk of disulfiram-like reaction during anidulafungin and metronidazole administration? 47th Annual Interscience

Conference on Antimicrobial Agents and Chemotherapy; September 17-20, 2007; Chicago, Illinois. Abstract K-2158.

Katiyar S, Pfaller M, Edlind T. Candida albicans and Candida glabrata clinical isolates exhibiting reduced echinocandin susceptibility. Antimicrob Agents Chemother. 2006;50:2892-2894.

Krause DS, Simjee AE, van Rensburg C, et al. A randomized, double-blind trial of anidulafungin versus fluconazole for the treatment of esophageal candidiasis. Clin Infect Dis. 2004a;39:770-775.

Krause DS, Reinhardt J, Vazquez JA, et al. Phase 2, randomized, dose-ranging study evaluating the safety and efficacy of anidulafungin in invasive candidiasis and candidemia. Antimicrob Agents Chemother. 2004b;48:2021-2024.

Kurtz MB, Rex JH. Glucan synthase inhibitors as antifungal agents. Adv Protein Chem. 2001;56:423-475.

Laverdiere M, Lalonde RG, Baril JG, Sheppard DC, Park S, Perlin DS. Progressive loss of echinocandin activity following prolonged use for treatment of Candida albicans oesophagitis. J Antimicrob Chemother. 2006;57:705-708.

Louie A, Deziel M, Liu W, Drusano MF, Gumbo T, Drusano GL. Pharmacodynamics of caspofungin in a murine model of systemic candidiasis: importance of persistence of caspofungin in tissues to understanding drug activity. Antimicrob Agents Chemother. 2005;49:5058-5068.

Maligie MA, Selitrennikoff CP. Cryptococcus neoformans resistance to echinocandins: $(1,3)$ beta-glucan synthase activity is sensitive to echinocandins. Antimicrob Agents Chemother. 2005;49:2851-2856.

Marco F, Pfaller MA, Messer SA, Jones RN. Activity of MK-0991 (L-743,872), a new echinocandin, compared with those of LY303366 and four other antifungal agents tested against blood stream isolates of Candida spp. Diagn Microbiol Infect Dis. 1998;32:33-37.

Miller CD, Lomaestro BW, Park S, Perlin DS. Progressive esophagitis caused by Candida albicans with reduced susceptibility to caspofungin. Pharmacother. 2006;26:877-880.

Moudgal V, Little T, Boikov D, Vazquez JA. Multiechinocandin- and multiazoleresistant Candida parapsilosis isolates serially obtained during therapy for prosthetic valve endocarditis. Antimicrob Agents Chemother. 2005;49:767-769.

Murdoch D, Plosker GL. Anidulafungin. Drugs. 2004;64:2249-2258.

Odabasi Z, Paetznick VL, Rodriguez JR, Chen E, Ostrosky-Zeichner L. In vitro activity of anidulafungin against selected clinically important mold isolates. Antimicrob Agents Chemother. 2004;48:1912-1915.

Osherov N, May GS, Albert ND, Kontoyiannis DP. Overexpression of Sbe2p, a Golgi protein, results in resistance to caspofungin in Saccharomyces cerevisiae. Antimicrob Agents Chemother. 2002;46:2462-2469.

Paderu P, Garcia-Effron G, Balashov S, Delmas G, Park S, Perlin DS. Serum differentially alters the antifungal properties of echinocandin drugs. Antimicrob Agents Chemother. 2007;51:2253-2256

Pappas PG, Rex JH, Sobel JD, Filler SG, Dismukes WE, Walsh TJ. Guidelines for treatment of candidiasis. Clin Infect Dis. 2004;38:161-189.

Park S, Kelly R, Kahn JN, et al. Specific substitutions in the echinocandin target Fks1p account for reduced susceptibility of rare laboratory and clinical Candida sp. isolates. Antimicrob Agents Chemother. 2005;49:3264-3273

Petraitiene R, Petraitis V, Groll AH, et al. Antifungal activity of LY303366, a novel echinocandin B, in experimental disseminated candidiasis in rabbits. Antimicrob Agents Chemother. 1999;43:2148-2155.
Pfaller MA, Boyken L, Hollis RJ, Messer SA, Tendolkar S, Diekema DJ. In vitro activities of anidulafungin against more than 2,500 clinical isolates of Candida spp., including 315 isolates resistant to fluconazole. $\underline{J \text { Clin Microbiol. }}$ 2005;43:5425-5427.

Pfaller MA, Boyken L, Hollis RJ, Messer SA, Tendolkar S, Diekema DJ. Global surveillance of in vitro activity of micafungin against Candida: a comparison with caspofungin by CLSI-recommended methods. J Clin Microbiol. 2006;44:3533-3538.

Pfaller MA. Anidulafungin: an echinocandin antifungal. Expert Opin Investig Drugs. 2004;13:1183-1197.

Philip A, Odabasi Z, Rodriguez JR, et al. In vitro synergy testing of anidulafungin with itraconazole, voriconazole, and amphotericin B against Aspergillus spp. and Fusarium spp. Antimicrob Agents Chemother. 2005;49:3572-3574.

Prescribing Information, Cancidas ${ }^{\circledR}$, available at: http://www.merck.com/product/usa/pi_circulars/c/cancidas/cancidas_pi.pdf

Prescribing Information, Eraxis ${ }^{\circledR}$, available at: http://www.pfizer.com/pfizer/download/uspi_eraxis.pdf

Prescribing Information, Mycamine ${ }^{\circledR}$, available at: http://www.astellas.us/docs/mycamine.pdf

Raasch RH. Anidulafungin: review of a new echinocandin antifungal agent. Expert Rev Anti Infect Ther. 2004;2:499-508.

Reboli AC, Rotstein C, Pappas PG, et al; Anidulafungin Study Group. Anidulafungin versus fluconazole for invasive candidiasis. N Engl J Med. 2007;356:2472-2482.

Tawara S, Ikeda F, Maki K, et al. In vitro activities of a new lipopeptide antifungal agent, FK463, against a variety of clinically important fungi. Antimicrob Agents Chemother. 2000;44:57-62.

Thye D, Kilfoil T, White R, Lasseter KC. Anidulafungin: pharmacokinetics in subjects with mild and moderate hepatic impairment. Presented at: 41st Interscience Conference on Antimicrobial Agents and Chemotherapy, 2001. Washington, DC. Abstract A34.

Uzun O, Kocagöz S, Cetinkaya Y, Arikan S, Unal S. In vitro activity of a new echinocandin, LY303366, compared with those of amphotericin B and fluconazole against clinical yeast isolates. Antimicrob Agents Chemother. 1997;41:1156-1157.

Vazquez JA, Lynch M, Boikov D, Sobel JD. In vitro activity of a new pneumocandin antifungal, L-743,872, against azole-susceptible and -resistant Candida species. Antimicrob Agents Chemother. 1997;41:1612-1614.

Vazquez JA. Anidulafungin: a new echinocandin with a novel profile. Clin Ther. 2005;27:657-673.

Vazquez JA. Anidulafungin: a novel echinocandin. Therapy. 2006a;3:39-54.

Vazquez JA. The safety of anidulafungin. Expert Opin Drug Saf. 2006b;5:751-758.

Vazquez JA, Sobel JD. Anidulafungin: a novel echinocandin. Clin Infect Dis. 2006;43:215-222.

Vazquez JA, Schranz J, Clark K, Goldstein B, Reboli A, Fichtenbaum C. A phase 2 , open-label study of the safety and efficacy of intravenous anidulafungin as a treatment for azole-refractory mucosal candidiasis. JAIDS. In press.

Wiederhold NP, Kontoyiannis DP, Chi J, Prince RA, Tam VH, Lewis RE. Pharmacodynamics of caspofungin in a murine model of invasive pulmonary aspergillosis: evidence of concentration-dependent activity. $\mathrm{J}$ Infect Dis. 2004;190:1464-1471.

Wiederhold NP, Lewis RE. The echinocandin antifungals: an overview of the pharmacology, spectrum and clinical efficacy. Expert Opin Investig Drugs. 2003;12:1313-1333.

Wisplinghoff $\mathrm{H}$, Bischoff T, Tallent SM, Seifert $\mathrm{H}$, Wenzel RP, Edmond MB. Nosocomial bloodstream infections in US hospitals: analysis of 24,179 cases from a prospective nationwide surveillance study. Clin Infect Dis. 2004;39:309-317.

Zaoutis TE, Argon J, Chu J, Berlin JA, Walsh TJ, Feudtner C. The epidemiology and attributable outcomes of candidemia in adults and children hospitalized in the United States: a propensity analysis. Clin Infect Dis. 2005;41:1232-1239. 
Zhanel GG, Karlowsky JA, Harding GA, et al. In vitro activity of a new semisynthetic echinocandin, LY-303366, against systemic isolates of Candida species, Cryptococcus neoformans, Blastomyces dermatitidis, and Aspergillus species. Antimicrob Agents Chemother. 1997;41:863-865.
Correspondence: Jose A. Vazquez, Henry Ford Hospital, Senior Staff, Professor of Medicine, Wayne State University School of Medicine, 2799 West Grand Blvd, CFP 202, Detroit, MI 48202, USA or at jvazque1@hfhs.org 